\title{
B3
}

\author{
doi: $10.14232 /$ fgykf.2018.b3
}

\section{Synthesis and evaluation cytotoxic and antioxidant effects of naringenin oxime relative to naringenin on human cancer cell lines}

\author{
Ahmed Latif, ${ }^{1,2}$ Tímea Gonda, ${ }^{1}$ Norbert Kúsz, ${ }^{1}$ Ágnes Kulmány, ${ }^{2}$ István Zupkó, ${ }^{2}$ \\ Attila Hunyadi ${ }^{1}$ \\ ${ }_{1}^{1}$ University of Szeged, Department of Pharmacognosy, 6720 Szeged, Eötvös u. 6. \\ 2 University of Szeged, Department of Pharmacodynamics and Biopharmacy, 670 Szeged, Eötvös u. 6.
}

A natural predominant flavanone naringenin that extracted from citrus fruits (grapefruit, orange) and tomato skin, has a wide range of pharmacological activities, including anti-inflammatory, antioxidant and anticancer effects. It is reported that naringenin exerts cytotoxic and apoptotic effects in several cancer cell line in a dose dependent manner, and when used in cancer chemotherapy ${ }^{(1-3)}$. The aim of the current study was the development of new derivatives with increased anticancer potency. Seven naringenin oxime derivatives, including four new derivatives, were synthesized. The structure of compounds was elucidated by NMR $\left({ }^{1} \mathrm{H},{ }^{13} \mathrm{C}\right)$ and HRMS assay. The cytotoxic and antioxidant effects of the synthetic compounds were investigated and compared to those elicited by naringenin. The antioxidant effects of the prepared compounds were demonstrated by means of ORAC and DPPH assays while antiproliferative properties were determined by MTT method against human cervical carcinoma (Hela, SiHa), breast carcinoma (MCF-7, MDA-MB-231) and leukemia (HL-60) cell lines.

The naringenin tert-butyl oxime demonstrated higher antiproliferative activity than parent and other synthetic derivatives, its calculated I $\mathrm{C}_{50}$ values were 19.5 and $23.5 \mu \mathrm{M}$ against MCF-7 and Hela cell lines, respectively. This compound was also induced apoptosis in Hela cells through the activation of caspase-3, and significant increase in the hypodiploid (sub G1) population as evidenced by cell cycle analysis.

\section{Acknowledgements:}

This work was supported by the National Research, Development and Innovation Office, Hungary (NKFIH; K119770 and K109293). A.H. acknowledges the János Bolyai fellowship of the Hungarian Academy of Sciences and the Kálmán Szász Prize.

\section{References:}

(1) P. Jong-Hwa, et al. J. Food Sci.Biotechnol. (2010), 19(3): 717-724.

(2) B. Lei, et al. J. Tumor Biol. (2016), 37: 11365-11374.

(3) W. Kaihua, et al. J. Clinical and Experimental pharmacology and physiology. (2017), 44: 862-871.

Supervisors: István Zupkó, Attila Hunyadi 